\title{
MITIGATION IN PACKET LOSS IN MANET BY INTRODUCING 2ACK BASED SCHEME WITH SORTING ALGORITHM
}

\author{
Naresh Kumar ${ }^{1}$
}

\begin{abstract}
MANET is a collection of mobile nodes that are capable of communicating each other via a wireless link. MANET can form at any place (no predefined infrastructure is needed) even on the fly or on the mountain or dense forest. There are certain parameters those are related to the performance of the MANET i.e. throughput, packet loss, delay etc. Throughput is directly depended upon the packet loss, if packet loss is high then throughput is low, so for the better performance of any MANET reduction in packet loss is very necessary. In this paper, we proposed a method which mitigates the packet loss by introducing a 2ACK scheme with sorting algorithm.
\end{abstract}

Keywords Mobile Ad hoc Network (MANET), AODV, Packet loss, 2ACK based approach.

\section{INTRODUCTION}

Mobile ad hoc network can be considered as a graph $\mathrm{G}=(\mathrm{N}, \mathrm{L})$, where $\mathrm{N}$ is the number of mobile nodes those are free to move and $\mathrm{L}$ is a set of bidirectional links [1]. The nodes can communicate dynamically without predefined infrastructure in arbitrary manner [2]. This flexible nature of MANET helps to design a network anytime anywhere (even in the fly). MANET is fully dynamically distributed in nature, in which each node is fully depends on another node of the network for the communication. Each node act as a host as well as a router.

Each of the nodes may have one or more wireless links interface and they communicate each other via radio transmission range [7]. A MANET might consider of numerous home computing devices, like cellular phone, pads, laptop, mobiles and so on. A node can communicate directly to another node, if it is in the transmission range of the another node, otherwise communication take place through intermediate nodes. There are a number of parameters that can be considered for performance evaluation of a real network, (it means how well a network runs) such as availability, reliability, response time, utilization ,throughput and packet loss [7].

Packet loss is accepted as one of the most significant parameter to evaluate the performance of the mobile ad hoc network. Due to highly dynamic topology, MANET transmission is highly error prone. Mobility, congestion, transmission error are the major cause that

${ }^{1}$ University Polytechnic, Jamia Millia Islamia, New Delhi-110025 
responsible for the packet loss in MANET [4]. These issues are directly associated with the network context (e.g. node mobility, load of the traffic). Congestion or bottleneck in a network occur when demand of a host cross the maximum limit of the communication link.

All the packets that has drop due to the mobility, congestion, transmission error and the attack is called the packet loss. Hence,

\section{Packet loss =sent packet - receive packet}

Besides of issues those discussed above, MANET suffered from some other serious issues like black hole attack, malicious attack and worm hole attack that are too responsible for the packet drop. Some nodes intentionally drop the packet, these nodes are called malicious nodes. Even after the optimal selection of the route, network can't perform well due to the packet drop [5]. Nodes are responsible for packet drop in two ways [4].

- $\quad$ nodes are discarding the packets due to insufficient resources (congestion).

- $\quad$ nodes are discarding the packet without any reason (malicious node).

Congestion also plays an important role in packet loss. Sometime buffer is overflow and it does not have enough space to accommodate the new incoming packets. But in another case node is discarding the packets due to the malicious node, these nodes uses the resources of the network but they are not willing to forward the packets to the next node. The presence of the malicious nodes makes negative impact on the performance of the network, malicious nodes increases packet loss in the network, If packet loss increases in the network, throughput decreases.

Link failure is another major cause of the packet loss in AODV and degrades the network performance. The network is consisting of a number of host, one is source host (a node which want to communicate) and another is destination host, and the nodes those are in between these two nodes is called intermediate nodes. There is an active node which is responsible for the routing information. New routes needed if source node, destination node or any one intermediate node moves or switch from its position [6].

\section{LAYERS RESPONSIBLE FOR PACKET LOSS}

Mainly MAC layer and network layer are responsible for all kind of packet losses. MAC layer is responsible for mobility related packet loss as well as congestion related packet loss, where as network layer is responsible only for mobility related packet loss in routing protocol.

Network layer When a packet reach at the network layer, routing protocol is responsible to forward the packet, if a route to the destination is available. Packet is prefer to buffer until a route is available.

Two cases of packet drop at network layer

1. If the buffer is overflow, when incoming packet needs to be buffered.

2. If the stored packet in the buffer exceed the expiration time (for AODV ns2 allows 30 sec time to live).

MAC layer MANET consists of a number of nodes, some nodes are within the range of the each other while some are out of the transmission range. In the MAC layer, mobility related packet loss occur when the next hop of the packet is out of the coverage area at the moment the packet is sent by the MAC layer protocol. This type of packet loss is mostly occurring in 
highly dynamic topology network. CSMA/CA protocol is responsible for the congestion related packet loss in MAC layer. The reason is that

1. The back off time is very high and it exceeds the limit.

2. Buffer is full (space is not available for new incoming packet).

As MANET is highly dynamic in nature due to its unique behavior of mobility characteristics; this behavior tends to link breakage in MANET. Due to the link breakage existing route became inactive and node discover new route by route request packet. RREQ messages are sent by the sender when a sender node wants to communicate and it is discovering a new route in on demand routing protocol, such as AODV evidential, mobility may increase the number of route request packet on the network.

Table 1 Packet loss at MAC and Network layer

\begin{tabular}{|l|l|l|}
\hline & MAC layer & $\begin{array}{l}\text { Network } \\
\text { layer }\end{array}$ \\
\hline $\begin{array}{l}\text { Mobility } \\
\text { related }\end{array}$ & Yes & Yes \\
\hline $\begin{array}{l}\text { Congestion } \\
\text { related }\end{array}$ & No & Yes \\
\hline
\end{tabular}

Total packet loss $=($ MAC layer loss $)+($ Network layer loss $)$

\section{RELATED WORK}

There has been a lot of work done on addressing packet loss in MANET. Yi Lu and Yuhui Zhong proposed a method to understand the main issues those are directly related to packet loss and showed that AODV has more packet loss due to the mobility as compare to congestion; hence AODV is more sensitive for mobility. Shalini Sharma et.al, proposed a method of secure channel to remove the problem of ambiguity and authentication which helps to minimize overhead and packets drop problem. Shiv shakti et.al., proposed method in which they used mix features of static as well as dynamic routing algorithm. The proposed system is capable enough to find out the next node for delivery of the packet. The proposed system checks the traffic density by calculating the ratio of incoming verses outgoing packet and compute the traffic density, and then decide whether to send the packet or not. It reduces the possibility of packet loss. Zhu qiankum et.al, implemented new advance AODV and observed the performance matrices such as network load packet drop, cost and delay for both normal AODV and the improved advance AODV and find out that advance AODV performs well and gives better throughput level with less delay and consume less energy. In advance AODV, packet loss is very less as compare to AODV. Hemant kumar et.al, proposed a method in which they introduced a method to identifie the broken links between two nodes, route can be repaired or can be discarded to avoid the packet loss. The proposed method can prevent the packet loss due to the traffic density and avoids from the malicious links.

\section{MODIFIED ACK BASED SCHEME}

A number of ACK based algorithms is used to find out the malicious node in the network. In the existing ACK based scheme each node sends ACK to the sender (in AODV, typically destination sends the acknowledgement to the sender). This acknowledgement based scheme produces a more number of ACK packets in the network. In other words it increases overhead in the network and the bulk amount of ACK is responsible to degrade the network performance. Now we modified the scheme by introducing sorting algorithm at the sender side. Every node is sending ACK to its sender after forwarding packets to the neighbor .It 
may possible that two or more than two consecutive nodes may have same number of ACK. Sorting process at the sender side decreases the overhead and eliminate the problem of due to the large overhead.

\section{PROPOSED ALGORITHM}

\section{/* Determination of malicious node */}

For each node determine the loss packets

If packet dropped > threshold

add the nodes into malicious node list

if (each node is suspected)

\{

node $\mathrm{X}$ checks $\mathrm{ACK}$ and sort them // $\mathrm{X}$ is the malicious listed node

if ( any ACK is missing from neighbor nodes)

\{

check the ACK from destination

comp( ACKneighbor, ACKdestination)

\{

if (( ACK neighbor - ACKdestination $)>=7)$

Add node into malicious node list

else

break;

\}\}

Table 2: Simulation parameters

\begin{tabular}{|l|l|}
\hline Number of nodes & 50 \\
\hline Routing protocol & AODV \\
\hline Area & $1000 * 1000$ \\
\hline Packet size & 512 bytes \\
\hline Mobility speed & $0-20(\mathrm{~ms})$ \\
\hline Simulation time & 250 \\
\hline Max no of connection & 40 \\
\hline Tool & MATLAB \\
\hline
\end{tabular}
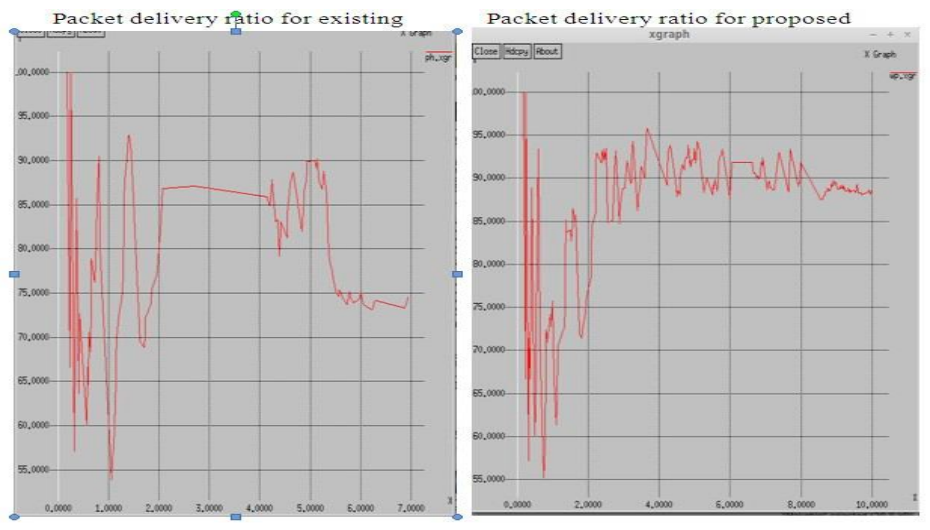

Figure 1 comparison in packet loss between existing method and proposed method 


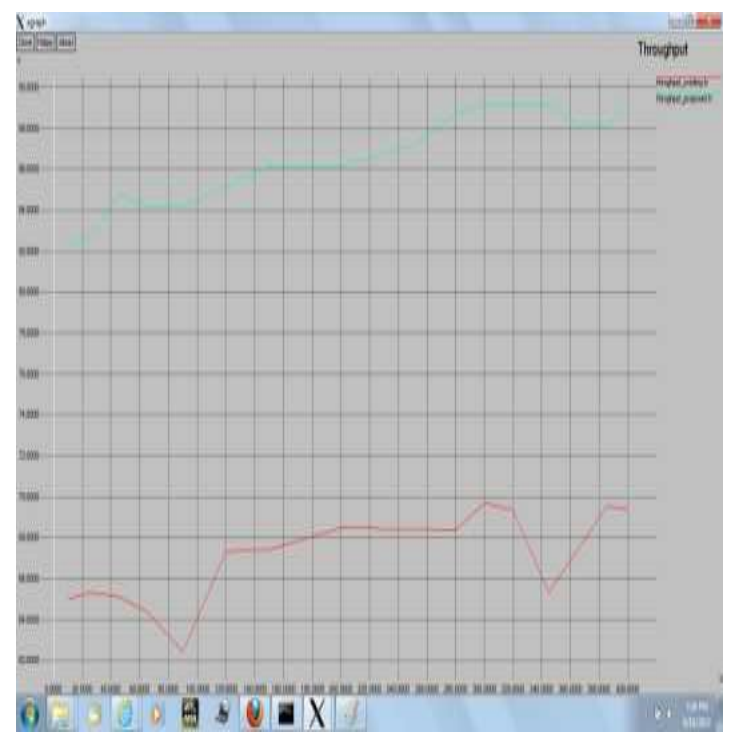

Figure 2 comparison in throughput between existing method and proposed method

\section{CONCLUSION}

It is concluded from the simulation result that throughput is directly effected by the packet loss in MANET. The two Ack based scheme with sorting algorithm minimise the overhead of the network and hence makes positive impact on the performance of the netwok.

\section{REFRENCES}

[1] Shobha tyagi et.al, "A Reliablity Based Variant of AODV In MANETs: Proposal, Analysis And Comparison" proceeding in $7^{\text {th }}$ international conference on communication, computing and virtualization, 2016.

[2] V. Bhanumati et.al, "RSS Based Energy EFFICIENT Scheme for the Reduction of Overhearing and Rebroadcast for MANET”, Elsevier, procedia engineering, vol. 38, 2463-2472, 2012.

[3] Zhu qiankun, "A mobile Ad Hoc Networks Algoritm Improved AODV Protocol,"in proceedings of international coneference on power electronics and engineering application, 2011.

[4] YiLu,et.al., "Packet Loss in Mobile Adhoc Networks", Computer science technical reports paper, Purdue University, 1558, 2003.

[5] ManishV.M et.al, " Diminution Of Packet Drop by Efficient Selection of Network Route in MANET"international journal of computer science and information technology" Vol 5(2), 2014.

[6] Anureet Kaur et.al., " Efficiency Enhancement in AOMDV to Reduce the Chances of Packet Loss in MANETs" in proceeding of International Journal of Science and Research( IJSR ), ISSN(on line):23197064, 2013.

[7] Saad Talib Hassan, "Designing a New MANET Environment using computer simulation" international journal of computer science and Electronics Engineering(IJCSEE) Volume 1,issue 3(2013)ISSN2320401x;EISSN 2320-4028, 2013.

[8] Pravin Ghosekar et.al., "Mobile Ad Hoc Networking:Imperatives and Challenges" proceedings in IJCA Special issues on "Mobile Ad-hoc Networks" (MANET), 2010.

[9] Shalini Sharma et.al., "Reducing Packet Loss in MANET"proceeding in networkand complex system ISSN 2224-610X( paper)ISSN 2225-0603(online), 2013.

[10] Shiv ShaktiSrivastva et.al., "Minimization of the Packet Losses in MANETS based on both static and Dynamic Routing Protocols"published by International Journal of Computer Application(IJCA), 2011.

[11] Ashutosh Lanjewar et.al., "Optimizing Cost,Delay,Packet Loss and Network Load in AODV Routing Protocol"in proceeding of International Journal of Computer Science and Information Security;Vol 11 No 4, 2013.

[12] Hemant Kumar Garg et.al. "Minimization of Average Delay, Routing Load and Packet Loss Rate in AODV Routing Protocol'International Journal of Computer Application volume 44-No15, 2012. 
[13] Sonali Gaikwad and D.S. Adane, "Reduction in routing overhead using 2-Ack scheme and novel routing algorithm", IJETT, vol. 4, issue 8, pp. 3677-3681, August 2013.

[14] J Sandeep et.al,2015 "Efficient Packet Transmission and Energy Optimization in Military Operation Scenarios of MANET” Proceeding of Computer Science, pp.- 400-407, 2015. 